\title{
Radiation dosimetry of florbetapir F 18
}

\author{
Abhinay D Joshi $i^{1 *}$, Michael J Pontecorvo ${ }^{1}$, Lee Adler², Michael G Stabin ${ }^{3}$, Daniel M Skovronsky ${ }^{1,4}$, \\ Alan P Carpenter ${ }^{1}$, Mark A Mintun ${ }^{1}$ and Florbetapir F 18 study investigators
}

\begin{abstract}
Background: Florbetapir is one of several ${ }^{18} \mathrm{~F}$-labeled amyloid plaque imaging tracers for positron emission tomography (PET). As the bio-distribution and radiation dose of PET tracers in human research are important for estimating the relative risks and benefits, a study was conducted to obtain this information on florbetapir.

Methods: Nine cognitively normal subjects (six females and three males, age $58 \pm 10$ years, weight $81 \pm 17 \mathrm{~kg}$ ) received an intravenous bolus injection of $395 \pm 27.9 \mathrm{MBq}$ of florbetapir, and whole-body emission scans were performed over approximately $6 \mathrm{~h}$. Computed tomography scans were acquired for attenuation correction. Volumes of interest (VOIs) for source organs including the brain, liver, lung, heart wall, and vertebrae were defined on the PET images. The VOls of the gallbladder, urinary bladder, and large and small intestines were also defined. Using reference man organ volumes (ICRP 30), total activity was calculated per organ for each time point. The resultant time-activity curves (TACs) were fitted with constrained exponentials. Kinetic data were entered into OLINDA/EXM software to calculate dose estimates; the dynamic urinary bladder and ICRP $30 \mathrm{Gl}$ tract models were employed. The effective dose (ED) for each subject was estimated from the acquired data using the adult model.

Results: The mean ED determined for nine healthy volunteers was $18.60 \pm 4.26 \mu \mathrm{Sv} / \mathrm{MBq}$ or $6.88 \mathrm{mSv}$ for a $370-\mathrm{MBq}$ dose. The organs that received the highest radiation absorbed doses were the gallbladder, upper large intestine, small intestine, liver, and urinary bladder at $143.0 \pm 80.20,74.50 \pm 34.20,65.50 \pm 29.60,64.40 \pm 22.10$, and $27.10 \pm 11.70 \mu \mathrm{SV} / \mathrm{MBq}$, respectively.
\end{abstract}

Conclusions: The ED for florbetapir has been calculated for nine healthy volunteers. At a dose of $370 \mathrm{MBq}$ florbetapir, the total average ED is approximately $6.88 \mathrm{mSv}$.

\section{Background}

Accumulation of amyloid- $\beta(A \beta)$ fibrils in the form of amyloid plaques is a neuropathological requirement for definitive diagnosis [1]. Positron emission tomography (PET) tracers that bind to aggregated $A \beta$ peptides offer promise to directly assess fibrillar amyloid pathology in vivo $[2,3]$. The initial studies with ${ }^{11} \mathrm{C}$ Pittsburgh Compound B (PiB) were the first to clearly demonstrate the feasibility of this approach [4]. However, the 20-min half-life of ${ }^{11} \mathrm{C}$ limits the use of this molecular imaging ligand to specialized research centers. To address this issue, several amyloid imaging

\footnotetext{
*Correspondence: joshi@avidrp.com

'Avid Radiopharmaceuticals, Inc., 3711 Market Street, 7th Floor, Philadelphia, PA 19104, USA

Full list of author information is available at the end of the article
}

ligands that use the longer-lived ${ }^{18} \mathrm{~F}$ PET isotope have been developed [5].

One of these tracers is florbetapir F $18((E)-4(2-(6-$ (2-(2-(2- $\left[{ }^{18} \mathrm{~F}\right]$ fluoroehoxy)ethoxy)ethoxy)pyridine-3-yl) vinyl)- $N$-mathylbenzeamine, also known as ${ }^{18} \mathrm{~F}-\mathrm{AV}-45$. When applied at tracer concentrations, florbetapir labels $A \beta$ plaques in sections from patients with pathologically confirmed Alzheimer's disease (AD) [6], and PET human studies show increased tracer retention in the brains of those clinically diagnosed with AD [7]. A recently reported autopsy study demonstrated high correspondence of florbetapir PET images to histopathology $[8,9]$. The present study was carried out to assess the radiation dosimetry of florbetapir.

\section{每 Springer}

(C) 2014 Joshi et al.; licensee Springer. This is an open access article distributed under the terms of the Creative Commons Attribution License (http://creativecommons.org/licenses/by/2.0), which permits unrestricted use, distribution, and reproduction in any medium, provided the original work is properly cited. 


\section{Methods}

\section{Subject population}

Nine healthy, cognitively normal subjects (three males and six females) with mean age of $58 \pm 10$ years and mean weight of $81 \pm 17 \mathrm{~kg}$ were recruited. The study protocol was institutional review board (Biomed IRB, San Diego, CA)-approved, and written informed consent form was obtained from each subject enrolled.

\section{PET imaging}

A catheter was placed in an antecubital vein in the subject's arm for injecting the tracer. Subjects were positioned supine in a Biograph 40 TruePoint PET/CT scanner (Siemens Medical Systems, Malvern, PA, USA) with the arms at their sides. For the purpose of this study, a whole-body (WB) scan was defined as a total of seven bed positions from the subject's top of the head to mid-thighs. Subjects wore an abdominal binder during imaging to reduce respiratory motion between PET and computed tomography (CT) acquisitions. Once positioned, a low-dose whole-body CT scan was obtained and then the subjects were injected with a target dose of $370 \mathrm{MBq}$ florbetapir (actual mean dose $395 \pm 27.9 \mathrm{MBq}$ ). Following injection, a session of up to four WB PET emission scans was acquired using the initial CT without subject repositioning. Following a short break (<30 min), a second low-dose CT was taken followed immediately by a session of at least two WB PET emission scans. Additional sessions consisting of low-dose CT and PET emission scans were subsequently obtained at roughly 60-min intervals for a total of up to seven sessions with breaks between the sessions for patient comfort. Each session included at least one PET whole-body emission scan (seven bed positions). The time per PET bed position was varied from $90 \mathrm{~s}$ for the first session to $4 \mathrm{~min}$ for the final session to compensate for radioactive decay.

In general, session 1 consisted of four PET scans, session 2 consisted of two PET scans, and sessions 3, 4, 5, 6 , and 7 consisted of one PET scan each. Although scan data was obtained for an average of approximately $6 \mathrm{~h}$ (range 4.25 to $7.37 \mathrm{~h}$ ) for all subjects, to accommodate subject comfort, some scans were omitted in some subjects: For four of nine subjects, all 11 emission scans were acquired; in three subjects, 10 emission scans were acquired; in one subject, 9 emission scans were acquired; and in one subject, the data were acquired in 7 scans. The time required to reposition the bed to initiate subsequent emission scans in a session was variable, and patients were given periodic breaks as requested. This resulted in variable start time across the subjects and variable number of image sequences (Table 1). The mean time for all subjects until the end of the last scan was $5.95 \pm 1.09 \mathrm{~h}$.
Table 1 CT and PET acquisition time sequence for all nine volunteers: frame start time after injection in minutes

\begin{tabular}{|c|c|c|c|c|c|c|c|c|c|}
\hline \multirow[t]{2}{*}{ Session } & \multicolumn{9}{|c|}{ Subject number } \\
\hline & 009 & 001 & 012 & 007 & 008 & 010 & 002 & 006 & 003 \\
\hline \multicolumn{10}{|c|}{ Low-dose CT scan after positioning and before florbetapir injection } \\
\hline 1.1 & 4 & 9 & 3 & 3 & 4 & 4 & 1 & 3 & 2 \\
\hline 1.2 & 18 & 25 & 16 & 20 & 17 & 22 & 18 & 15 & 20 \\
\hline 1.3 & 29 & 50 & 30 & 33 & 30 & 39 & NA & 26 & 37 \\
\hline 1.4 & 47 & 64 & 45 & 46 & 46 & 55 & 52 & 39 & NA \\
\hline \multicolumn{10}{|c|}{ Short break <30 min } \\
\hline \multicolumn{10}{|c|}{ Low-dose CT scan after positioning } \\
\hline 2.1 & 76 & 105 & 71 & 85 & 72 & 90 & 88 & 64 & 76 \\
\hline 2.2 & 94 & 120 & 88 & 102 & 89 & 109 & 113 & 79 & 102 \\
\hline 2.3 & NA & NA & NA & NA & NA & NA & 133 & NA & NA \\
\hline \multicolumn{10}{|c|}{ Low-dose CT scan after positioning } \\
\hline 3.1 & 137 & 170 & 129 & 129 & 130 & 187 & 206 & 112 & 153 \\
\hline \multicolumn{10}{|c|}{ Low-dose CT scan after positioning } \\
\hline 4.1 & 204 & 236 & 198 & 180 & 186 & 246 & 250 & 181 & 228 \\
\hline \multicolumn{10}{|c|}{ Low-dose CT scan after positioning } \\
\hline 5.1 & 268 & 301 & 241 & 248 & 246 & 306 & 303 & 240 & NA \\
\hline \multicolumn{10}{|c|}{ Low-dose CT scan after positioning } \\
\hline 6.1 & 326 & 363 & NA & NA & 308 & 366 & 364 & 301 & NA \\
\hline \multicolumn{10}{|c|}{ Low-dose CT scan after positioning } \\
\hline 7.1 & 384 & 428 & NA & 360 & 356 & NA & NA & 361 & NA \\
\hline
\end{tabular}

NA means that the subject is not imaged during that duration of the session. PET scans are identified as session\#.scan\#.

\section{PET data post-processing}

The acquired emission data were reconstructed using iterative reconstruction (two iterations, eight subsets), with attenuation, random, and scatter correction, and a post-reconstruction Gaussian filter of $7 \mathrm{~mm}$ was applied. PET whole-body images were generated with a matrix size of $128 \times 128$ per slice and a voxel size of $5.35 \times 5.35 \times 3 \mathrm{~mm}^{3}$. The camera sensitivity was calibrated using a uniform cylindrical phantom (dimensions: $20-\mathrm{cm}$ diameter, 30-cm length, and 9,420-mL volume) filled with a known concentration of activity to generate a camera-to-well counter cross-calibration factor (approximately $1.1(\mathrm{~Bq} / \mathrm{mL}) / \mathrm{cps})$ ) which was subsequently applied to all PET measurement of in vivo activity.

\section{Dosimetry analysis}

Following reconstruction, volumes of interest (VOIs) for various body organs were defined on the PET images for each patient. Based on the visual examination, the anatomical information from the CT data and the human whole-body reference atlas $[10,11]$ was used to 

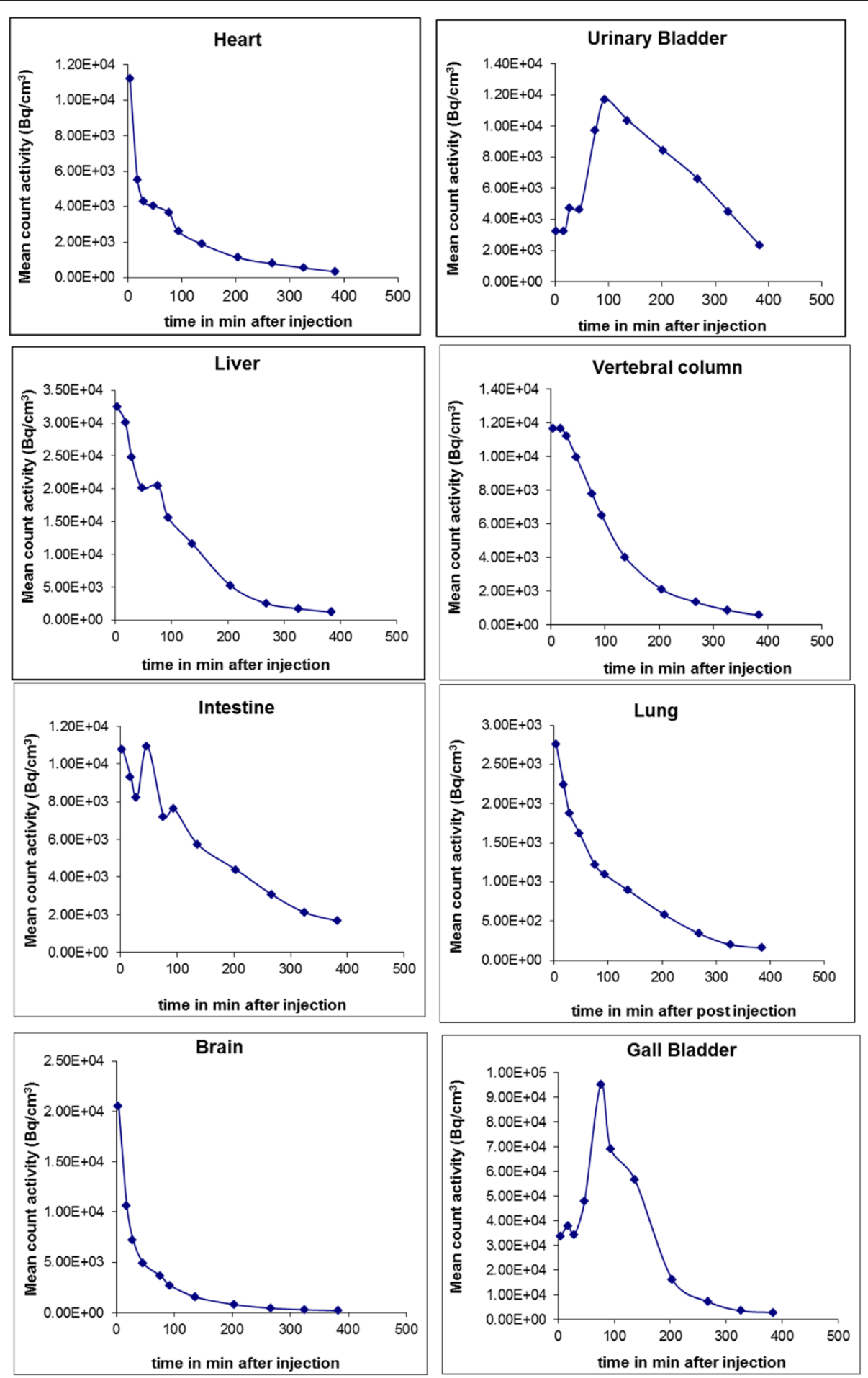

Figure 1 Time-activity curves showing mean count activity $\left(\mathrm{Bq} / \mathrm{cm}^{3}\right)$ over time in eight organs for a representative subject. Mean radioactivity concentration values are different across the organs, so the upper limit of the scale is variable.

delineate patient specific VOIs. Organs defined by the VOI process were the brain, liver, lung, heart wall, urinary bladder, vertebrae, intestine, and gallbladder.
Using these regions on the PET emission data, average counts for the organs were determined at each time imaged. The regional activities were then calculated for 
each time point, thus providing a time-activity curve (TAC) for each region of a given subject (Figure 1). All organ TACs were fitted with one or two exponential terms using SAAM II software [12]. Time integrals of activity for all the organs were calculated from a lower limit of zero to an upper limit of infinity and expressed as the number of disintegrations in source organs [13]. These values were entered in OLINDA/EXM software [14], using the adult model. Activity observed in a segment of the gastrointestinal (GI) tract was used to estimate total intestinal excretion, using the ICRP $30 \mathrm{GI}$ kinetic model in the OLINDA/EXM software.

The cumulated activity in the urinary bladder contents was determined by fitting the TAC of the bladder contents with an exponential in-growth function of the form:

$$
U(t)=U(0) \times\left(1-e^{-t / \tau}\right)
$$

where $U(t)$ is the fraction of activity in the bladder at time ' $t$ ' after injection and $\tau$ is the half-life of ${ }^{18} \mathrm{~F} / \mathrm{ln}[2]$. These parameters were entered into the voiding bladder model of OLINDA/EXM. For the principal analysis, no bladder voiding was assumed. A separate analysis was performed with no bladder void until 90 min post-injection.

Additional calculations were performed using the feature in OLINDA/EXM that allows modification of doses on body mass ratios to adjust the calculations to be more appropriate for the subject's individual body size. All organs and the whole body were selected and scaled by ratios of the subject's body weight to that of the reference phantom to develop dose estimates for $50-, 60-$, and $80-\mathrm{kg}$ individuals (the reference adult is approximately $70 \mathrm{~kg}$ ).

\section{Results}

Figure 2 shows a typical series of PET scans in a healthy volunteer enrolled in this study. The series of images demonstrates rapid distribution of florbetapir shortly following injection. Essentially, immediate uptake into, followed by rapid clearance out of, the normal brain was seen. Rapid clearance from circulation and localization in the liver was also observed. Some accumulation was observed in the urinary bladder, but primarily, the elimination appeared to occur by way of clearance from the liver and excretion through the gallbladder into the GI tract.

Cumulated activities in each organ were determined for individual subjects to allow subject-specific dose estimates to be determined. Based on the nine subject measurements of florbetapir, the effective dose (ED) was determined to be $18.60 \pm 4.260 \mu \mathrm{Sv} / \mathrm{MBq}$ or $6.88 \mathrm{mSv}$ for a $370-\mathrm{MBq}$ dose with no specific assumptions regarding bladder voiding. In a separate analysis assuming no urinary bladder voiding until $90 \mathrm{~min}$, the ED was

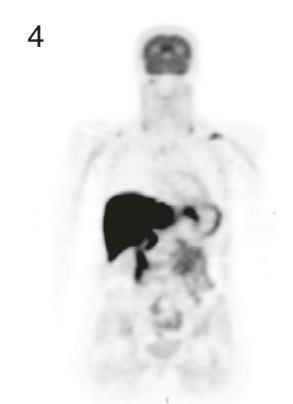

130

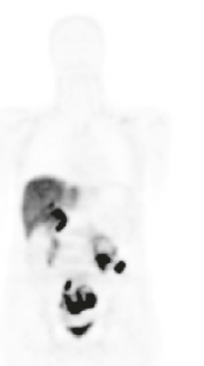

186

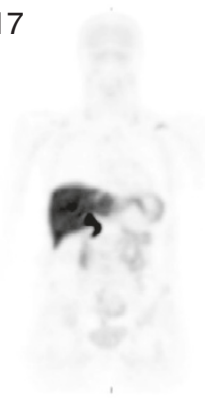

30

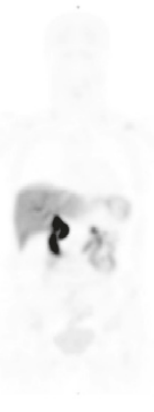

246
46

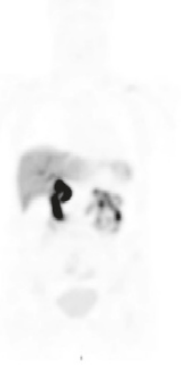

72

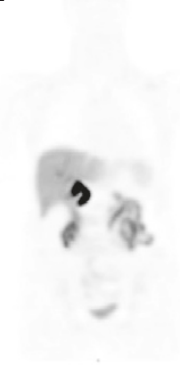

308
89
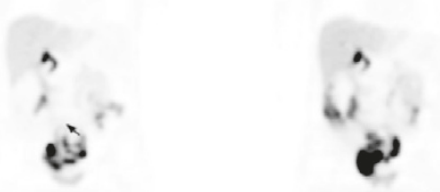

356

Figure 2 Series of PET whole-body images from 4 to $356 \mathrm{~min}$ after injection of florbetapir F 18. 
Table 2 Radiation dose estimates ( $\mu \mathrm{Sv} / \mathrm{MBq}$ ) with no urinary bladder voiding

\begin{tabular}{|c|c|c|c|c|c|c|c|c|c|c|c|}
\hline Target organ & 024-009 & $024-001$ & 024-012 & 024-007 & 024-008 & 024-010 & 024-002 & 024-006 & 024-003 & Average & Std Dev \\
\hline Adrenals & $1.30 E+01$ & $1.24 \mathrm{E}+01$ & $1.63 \mathrm{E}+01$ & $1.34 \mathrm{E}+01$ & $1.42 \mathrm{E}+01$ & $1.40 \mathrm{E}+01$ & $1.33 E+01$ & $1.38 \mathrm{E}+01$ & $1.17 E+01$ & $1.36 \mathrm{E}+01$ & $1.29 \mathrm{E}+00$ \\
\hline Brain & $1.27 E+01$ & $7.03 E+00$ & $9.50 \mathrm{E}+00$ & $8.25 E+00$ & $1.12 \mathrm{E}+01$ & $1.20 \mathrm{E}+01$ & $1.10 E+01$ & $9.86 \mathrm{E}+00$ & $8.66 \mathrm{E}+00$ & $1.00 \mathrm{E}+01$ & $1.86 \mathrm{E}+00$ \\
\hline Breasts & $6.76 \mathrm{E}+00$ & $6.87 E+00$ & $6.66 \mathrm{E}+00$ & $6.98 \mathrm{E}+00$ & $4.61 E+00$ & $6.73 E+00$ & $5.09 E+00$ & $5.78 \mathrm{E}+00$ & $6.65 E+00$ & $6.24 \mathrm{E}+00$ & $8.65 E-01$ \\
\hline Gallbladder wall & $7.18 \mathrm{E}+01$ & $8.30 E+01$ & $2.22 \mathrm{E}+02$ & $1.26 \mathrm{E}+02$ & $2.00 E+02$ & $8.36 \mathrm{E}+01$ & $1.07 E+02$ & $3.02 E+02$ & $8.87 E+01$ & $1.43 E+02$ & $8.02 \mathrm{E}+01$ \\
\hline LLI wall & $3.05 E+01$ & $2.09 E+01$ & $2.19 E+01$ & $3.37 E+01$ & $4.16 E+01$ & $2.90 E+01$ & $4.21 E+01$ & $1.57 \mathrm{E}+01$ & $1.51 E+01$ & $2.78 \mathrm{E}+01$ & $1.02 E+01$ \\
\hline Small intestine & $7.08 \mathrm{E}+01$ & $4.36 E+01$ & $5.05 E+01$ & $7.91 E+01$ & $1.10 E+02$ & $6.69 E+01$ & $1.07 E+02$ & $3.47 E+01$ & $2.73 E+01$ & $6.55 E+01$ & $2.96 \mathrm{E}+01$ \\
\hline Stomach wall & $1.22 \mathrm{E}+01$ & $1.13 E+01$ & $1.22 \mathrm{E}+01$ & $1.31 E+01$ & $1.17 E+01$ & $1.22 \mathrm{E}+01$ & $1.19 E+01$ & $1.04 \mathrm{E}+01$ & $1.03 E+01$ & $1.17 E+01$ & $9.06 \mathrm{E}-01$ \\
\hline ULI wall & $7.96 \mathrm{E}+01$ & $4.86 E+01$ & $5.79 E+01$ & $8.93 E+01$ & $1.27 E+02$ & $7.56 \mathrm{E}+01$ & $1.22 E+02$ & $4.02 E+01$ & $3.00 E+01$ & $7.45 E+01$ & $3.42 E+01$ \\
\hline Heart wall & $1.35 E+01$ & $1.06 E+01$ & $1.41 E+01$ & $1.18 \mathrm{E}+01$ & $1.55 E+01$ & $1.46 \mathrm{E}+01$ & $1.35 E+01$ & $1.27 \mathrm{E}+01$ & $1.14 \mathrm{E}+01$ & $1.31 \mathrm{E}+01$ & $1.59 \mathrm{E}+00$ \\
\hline Kidneys & $1.28 \mathrm{E}+01$ & $1.19 E+01$ & $1.46 E+01$ & $1.37 E+01$ & $1.38 \mathrm{E}+01$ & $1.33 E+01$ & $1.33 E+01$ & $1.24 \mathrm{E}+01$ & $1.09 \mathrm{E}+01$ & $1.30 \mathrm{E}+01$ & $1.11 E+00$ \\
\hline Liver & $4.63 E+01$ & $4.18 \mathrm{E}+01$ & $9.81 E+01$ & $4.81 E+01$ & $9.25 E+01$ & $6.42 E+01$ & $7.57 E+01$ & $7.41 E+01$ & $3.89 E+01$ & $6.44 \mathrm{E}+01$ & $2.21 E+01$ \\
\hline Lungs & $8.54 \mathrm{E}+00$ & $7.69 E+00$ & $9.37 \mathrm{E}+00$ & $7.67 \mathrm{E}+00$ & $9.34 \mathrm{E}+00$ & $9.31 E+00$ & $8.14 \mathrm{E}+00$ & $9.19 \mathrm{E}+00$ & $7.47 \mathrm{E}+00$ & $8.52 \mathrm{E}+00$ & $8.01 E-01$ \\
\hline Muscle & $9.36 \mathrm{E}+00$ & $8.87 E+00$ & $8.70 E+00$ & $9.77 E+00$ & $7.60 E+00$ & $9.19 E+00$ & $8.23 E+00$ & $7.56 \mathrm{E}+00$ & $8.36 \mathrm{E}+00$ & $8.63 E+00$ & $7.62 E-01$ \\
\hline Ovaries & $1.93 E+01$ & $1.50 E+01$ & $1.50 E+01$ & $2.11 E+01$ & $2.24 \mathrm{E}+01$ & $1.86 \mathrm{E}+01$ & $2.34 \mathrm{E}+01$ & $1.16 E+01$ & $1.23 E+01$ & $1.76 E+01$ & $4.34 \mathrm{E}+00$ \\
\hline Pancreas & $1.37 E+01$ & $1.31 E+01$ & $1.70 E+01$ & $1.47 E+01$ & $1.51 E+01$ & $1.45 \mathrm{E}+01$ & $1.40 E+01$ & $1.50 \mathrm{E}+01$ & $1.23 E+01$ & $1.44 \mathrm{E}+01$ & $1.34 \mathrm{E}+00$ \\
\hline Red marrow & $1.67 E+01$ & $1.26 \mathrm{E}+01$ & $1.33 \mathrm{E}+01$ & $1.44 \mathrm{E}+01$ & $1.66 \mathrm{E}+01$ & $1.51 E+01$ & $1.56 \mathrm{E}+01$ & $1.28 \mathrm{E}+01$ & $1.19 E+01$ & $1.43 \mathrm{E}+01$ & $1.78 \mathrm{E}+00$ \\
\hline Osteogenic cells & $3.43 E+01$ & $2.43 E+01$ & $2.43 E+01$ & $2.60 \mathrm{E}+01$ & $3.19 E+01$ & $2.94 \mathrm{E}+01$ & $2.88 \mathrm{E}+01$ & $2.58 \mathrm{E}+01$ & $2.38 \mathrm{E}+01$ & $2.76 \mathrm{E}+01$ & $3.71 E+00$ \\
\hline Skin & $6.62 E+00$ & $6.51 E+00$ & $5.97 E+00$ & $6.79 E+00$ & $4.47 E+00$ & $6.41 E+00$ & $5.08 \mathrm{E}+00$ & $5.23 E+00$ & $6.25 E+00$ & $5.93 E+00$ & $8.09 E-01$ \\
\hline Spleen & $9.73 E+00$ & $9.53 E+00$ & $9.06 \mathrm{E}+00$ & $1.02 E+01$ & $7.28 \mathrm{E}+00$ & $9.51 E+00$ & $7.96 \mathrm{E}+00$ & $7.86 \mathrm{E}+00$ & $8.99 \mathrm{E}+00$ & $8.90 \mathrm{E}+00$ & $9.86 \mathrm{E}-01$ \\
\hline Testes & $7.75 E+00$ & $7.84 \mathrm{E}+00$ & $6.44 E+00$ & $8.20 E+00$ & $4.37 E+00$ & $7.51 E+00$ & $5.93 E+00$ & $5.62 E+00$ & $7.66 \mathrm{E}+00$ & $6.81 E+00$ & $1.29 E+00$ \\
\hline Thymus & $8.11 E+00$ & $8.26 E+00$ & $7.51 E+00$ & $8.30 E+00$ & $4.97 E+00$ & $7.93 \mathrm{E}+00$ & $5.73 E+00$ & $6.59 E+00$ & $8.04 E+00$ & $7.27 E+00$ & $1.22 \mathrm{E}+00$ \\
\hline Thyroid & $7.90 E+00$ & $7.98 \mathrm{E}+00$ & $6.61 E+00$ & $7.93 E+00$ & $4.09 E+00$ & $7.43 E+00$ & $5.06 \mathrm{E}+00$ & $5.96 \mathrm{E}+00$ & $7.81 E+00$ & $6.75 E+00$ & $1.43 \mathrm{E}+00$ \\
\hline Urinary bladder wall & $2.25 E+01$ & $1.76 E+01$ & $2.03 E+01$ & $2.89 E+01$ & $2.64 E+01$ & $3.29 E+01$ & $5.47 E+01$ & $1.56 \mathrm{E}+01$ & $2.48 \mathrm{E}+01$ & $2.71 E+01$ & $1.17 E+01$ \\
\hline Uterus & $1.68 \mathrm{E}+01$ & $1.38 \mathrm{E}+01$ & $1.35 E+01$ & $1.85 E+01$ & $1.80 E+01$ & $1.67 E+01$ & $2.03 E+01$ & $1.08 \mathrm{E}+01$ & $1.22 \mathrm{E}+01$ & $1.56 \mathrm{E}+01$ & $3.19 \mathrm{E}+00$ \\
\hline Total body & $1.22 \mathrm{E}+01$ & $1.07 E+01$ & $1.22 \mathrm{E}+01$ & $1.22 \mathrm{E}+01$ & $1.23 E+01$ & $1.22 \mathrm{E}+01$ & $1.22 \mathrm{E}+01$ & $1.04 \mathrm{E}+01$ & $9.96 \mathrm{E}+00$ & $1.16 \mathrm{E}+01$ & $9.51 E-01$ \\
\hline Effective dose & $1.90 \mathrm{E}+01$ & $1.49 \mathrm{E}+01$ & $1.77 E+01$ & $2.01 E+01$ & $2.44 \mathrm{E}+01$ & $1.98 \mathrm{E}+01$ & $2.49 \mathrm{E}+01$ & $1.43 \mathrm{E}+01$ & $1.27 E+01$ & $1.86 \mathrm{E}+01$ & $4.26 \mathrm{E}+00$ \\
\hline
\end{tabular}

Std Dev, standard deviation; LLI, lower large intestine; ULI, upper large intestine. 
Table 3 Radiation dose estimates $(\mu \mathrm{Sv} / \mathrm{MBq})$ assuming one urinary bladder void at 90 min post-injection

\begin{tabular}{|c|c|c|c|c|c|c|c|c|c|c|c|}
\hline Target organ & 24-009 & $24-001$ & $24-012$ & $24-007$ & 24-008 & $24-010$ & $24-002$ & $24-006$ & 24-003 & Average & Std Dev \\
\hline Adrenals & $1.30 \mathrm{E}+01$ & $1.24 \mathrm{E}+01$ & $1.63 E+01$ & $1.34 \mathrm{E}+01$ & $1.42 \mathrm{E}+01$ & $1.40 \mathrm{E}+01$ & $1.33 E+01$ & $1.38 \mathrm{E}+01$ & $1.17 E+01$ & $1.36 \mathrm{E}+01$ & $1.29 E+00$ \\
\hline Brain & $1.27 E+01$ & $7.03 E+00$ & $9.50 E+00$ & $8.25 E+00$ & $1.12 \mathrm{E}+01$ & $1.20 \mathrm{E}+01$ & $1.10 E+01$ & $9.86 \mathrm{E}+00$ & $8.66 \mathrm{E}+00$ & $1.00 \mathrm{E}+01$ & $1.86 \mathrm{E}+00$ \\
\hline Breasts & $6.76 \mathrm{E}+00$ & $6.87 E+00$ & $6.66 E+00$ & $6.98 \mathrm{E}+00$ & $4.61 E+00$ & $6.73 E+00$ & $5.09 \mathrm{E}+00$ & $5.78 \mathrm{E}+00$ & $6.65 E+00$ & $6.24 E+00$ & $8.65 E-01$ \\
\hline Gallbladder wall & $7.18 \mathrm{E}+01$ & $8.30 E+01$ & $2.22 \mathrm{E}+02$ & $1.26 \mathrm{E}+02$ & $2.00 E+02$ & $8.35 E+01$ & $1.07 E+02$ & $3.02 E+02$ & $8.87 E+01$ & $1.43 E+02$ & $8.02 \mathrm{E}+01$ \\
\hline LLI wall & $3.03 E+01$ & $2.09 \mathrm{E}+01$ & $2.18 \mathrm{E}+01$ & $3.37 E+01$ & $4.15 E+01$ & $2.88 \mathrm{E}+01$ & $4.20 E+01$ & $1.57 E+01$ & $1.50 E+01$ & $2.77 E+01$ & $1.02 \mathrm{E}+01$ \\
\hline Small intestine & $7.07 E+01$ & $4.36 \mathrm{E}+01$ & $5.04 \mathrm{E}+01$ & $7.91 E+01$ & 1.10E + 02 & $6.69 E+01$ & $1.07 E+02$ & $3.47 \mathrm{E}+01$ & $2.73 E+01$ & $6.55 E+01$ & $2.96 \mathrm{E}+01$ \\
\hline Stomach wall & $1.22 \mathrm{E}+01$ & $1.13 E+01$ & $1.22 \mathrm{E}+01$ & $1.31 E+01$ & $1.17 E+01$ & $1.22 \mathrm{E}+01$ & $1.19 E+01$ & $1.03 E+01$ & $1.03 \mathrm{E}+01$ & $1.17 E+01$ & $9.24 \mathrm{E}-01$ \\
\hline ULI wall & $7.96 \mathrm{E}+01$ & $4.86 \mathrm{E}+01$ & $5.79 E+01$ & $8.93 E+01$ & $1.26 E+02$ & $7.55 E+01$ & $1.22 \mathrm{E}+02$ & $4.02 E+01$ & $3.00 E+01$ & $7.43 E+01$ & $3.40 E+01$ \\
\hline Heart wall & $1.35 E+01$ & $1.06 \mathrm{E}+01$ & $1.41 E+01$ & $1.18 \mathrm{E}+01$ & $1.55 E+01$ & $1.46 \mathrm{E}+01$ & $1.35 \mathrm{E}+01$ & $1.27 \mathrm{E}+01$ & $1.14 \mathrm{E}+01$ & $1.31 E+01$ & $1.59 \mathrm{E}+00$ \\
\hline Kidneys & $1.28 \mathrm{E}+01$ & $1.19 E+01$ & $1.46 \mathrm{E}+01$ & $1.37 E+01$ & $1.38 \mathrm{E}+01$ & $1.33 E+01$ & $1.33 E+01$ & $1.24 \mathrm{E}+01$ & $1.09 E+01$ & $1.30 E+01$ & $1.11 E+00$ \\
\hline Liver & $4.63 E+01$ & $4.18 \mathrm{E}+01$ & $9.81 E+01$ & $4.81 E+01$ & $9.25 E+01$ & $6.42 E+01$ & $7.57 \mathrm{E}+01$ & $7.41 E+01$ & $3.89 E+01$ & $6.44 \mathrm{E}+01$ & $2.21 E+01$ \\
\hline Lungs & $8.54 \mathrm{E}+00$ & $7.69 \mathrm{E}+00$ & $9.37 E+00$ & $7.67 E+00$ & $9.34 \mathrm{E}+00$ & $9.31 E+00$ & $8.14 \mathrm{E}+00$ & $9.18 \mathrm{E}+00$ & $7.47 E+00$ & $8.52 E+00$ & $7.99 \mathrm{E}-01$ \\
\hline Muscle & $9.31 E+00$ & $8.87 E+00$ & $8.68 \mathrm{E}+00$ & $9.78 \mathrm{E}+00$ & $7.57 E+00$ & $9.15 E+00$ & $8.22 E+00$ & $7.55 E+00$ & $8.33 E+00$ & $8.61 E+00$ & $7.63 E-01$ \\
\hline Ovaries & $1.91 E+01$ & $1.50 \mathrm{E}+01$ & $1.49 \mathrm{E}+01$ & $2.11 E+01$ & $2.23 E+01$ & $1.84 \mathrm{E}+01$ & $2.34 \mathrm{E}+01$ & $1.16 \mathrm{E}+01$ & $1.22 \mathrm{E}+01$ & $1.76 \mathrm{E}+01$ & $4.33 E+00$ \\
\hline Pancreas & $1.37 E+01$ & $1.31 E+01$ & $1.70 E+01$ & $1.47 E+01$ & $1.50 \mathrm{E}+01$ & $1.45 \mathrm{E}+01$ & $1.40 \mathrm{E}+01$ & $1.50 \mathrm{E}+01$ & $1.23 \mathrm{E}+01$ & $1.44 \mathrm{E}+01$ & $1.34 \mathrm{E}+00$ \\
\hline Red marrow & $1.67 E+01$ & $1.26 \mathrm{E}+01$ & $1.33 E+01$ & $1.44 E+01$ & $1.66 \mathrm{E}+01$ & $1.51 E+01$ & $1.56 E+01$ & $1.28 \mathrm{E}+01$ & $1.19 E+01$ & $1.43 \mathrm{E}+01$ & $1.78 \mathrm{E}+00$ \\
\hline Osteogenic cells & $3.43 E+01$ & $2.43 E+01$ & $2.43 E+01$ & $2.60 E+01$ & $3.19 E+01$ & $2.94 \mathrm{E}+01$ & $2.88 \mathrm{E}+01$ & $2.58 \mathrm{E}+01$ & $2.38 \mathrm{E}+01$ & $2.76 \mathrm{E}+01$ & $3.71 E+00$ \\
\hline Skin & $6.60 E+00$ & $6.51 E+00$ & $5.96 \mathrm{E}+00$ & $6.79 E+00$ & $4.46 E+00$ & $6.39 E+00$ & $5.08 \mathrm{E}+00$ & $5.23 \mathrm{E}+00$ & $6.24 \mathrm{E}+00$ & $5.92 \mathrm{E}+00$ & 8.07E- 01 \\
\hline Spleen & $9.72 \mathrm{E}+00$ & $9.53 \mathrm{E}+00$ & $9.06 \mathrm{E}+00$ & $1.03 E+01$ & $7.27 E+00$ & $9.50 \mathrm{E}+00$ & $7.96 \mathrm{E}+00$ & $7.86 \mathrm{E}+00$ & $8.98 \mathrm{E}+00$ & $8.91 E+00$ & $1.00 \mathrm{E}+00$ \\
\hline Testes & $7.62 E+00$ & $7.84 E+00$ & $6.37 E+00$ & $8.22 E+00$ & $4.29 E+00$ & $7.39 E+00$ & $5.89 E+00$ & $5.58 \mathrm{E}+00$ & $7.57 E+00$ & $6.75 E+00$ & $1.30 E+00$ \\
\hline Thymus & $8.11 E+00$ & $8.26 \mathrm{E}+00$ & $7.51 E+00$ & $8.30 E+00$ & $4.97 E+00$ & $7.93 E+00$ & $5.73 E+00$ & $6.59 E+00$ & $8.04 \mathrm{E}+00$ & $7.27 \mathrm{E}+00$ & $1.22 \mathrm{E}+00$ \\
\hline Thyroid & $7.90 \mathrm{E}+00$ & $7.98 \mathrm{E}+00$ & $6.61 E+00$ & $7.93 E+00$ & $4.09 E+00$ & $7.43 E+00$ & $5.06 \mathrm{E}+00$ & $5.96 \mathrm{E}+00$ & $7.81 E+00$ & $6.75 E+00$ & $1.43 E+00$ \\
\hline Urinary bladder wall & $1.54 \mathrm{E}+01$ & $1.76 E+01$ & $1.65 E+01$ & $2.98 \mathrm{E}+01$ & $2.21 E+01$ & $2.67 E+01$ & $5.28 \mathrm{E}+01$ & $1.38 \mathrm{E}+01$ & $2.01 E+01$ & $2.39 \mathrm{E}+01$ & $1.21 \mathrm{E}+01$ \\
\hline Uterus & $1.64 \mathrm{E}+01$ & $1.38 \mathrm{E}+01$ & $1.33 E+01$ & $1.86 \mathrm{E}+01$ & $1.78 \mathrm{E}+01$ & $1.63 \mathrm{E}+01$ & $2.02 \mathrm{E}+01$ & $1.06 \mathrm{E}+01$ & $1.19 \mathrm{E}+01$ & $1.54 \mathrm{E}+01$ & $3.22 \mathrm{E}+00$ \\
\hline Total body & $1.21 E+01$ & $1.07 \mathrm{E}+01$ & $1.22 E+01$ & $1.22 \mathrm{E}+01$ & $1.22 \mathrm{E}+01$ & $1.22 \mathrm{E}+01$ & $1.22 \mathrm{E}+01$ & $1.04 \mathrm{E}+01$ & $9.93 \mathrm{E}+00$ & $1.16 \mathrm{E}+01$ & $9.41 \mathrm{E}-01$ \\
\hline Effective dose & $1.86 \mathrm{E}+01$ & $1.49 \mathrm{E}+01$ & $1.75 E+01$ & $2.01 E+01$ & $2.41 E+01$ & $1.94 \mathrm{E}+01$ & $2.48 \mathrm{E}+01$ & $1.42 E+01$ & $1.25 \mathrm{E}+01$ & $1.85 \mathrm{E}+01$ & $4.23 E+00$ \\
\hline Adjusted bladder AUC & $1.10 E+01$ & $1.70 E+01$ & $1.70 E+01$ & $4.00 E+01$ & $3.00 \mathrm{E}+01$ & $3.60 E+01$ & $9.30 \mathrm{E}+01$ & $1.40 \mathrm{E}+01$ & $2.40 E+01$ & $3.13 E+01$ & $2.52 \mathrm{E}+01$ \\
\hline
\end{tabular}


Table 4 Average dose estimates ( $\mu \mathrm{Sv} / \mathrm{MBq}$ ) across nine healthy volunteers for $50-, 60-$, and $80-\mathrm{kg}$ scaled weights

\begin{tabular}{|c|c|c|c|c|c|c|c|c|}
\hline \multirow[t]{2}{*}{ Target organ } & \multicolumn{2}{|c|}{$50 \mathrm{~kg}$} & \multicolumn{2}{|c|}{$60 \mathrm{~kg}$} & \multicolumn{2}{|c|}{70 kg } & \multicolumn{2}{|c|}{$80 \mathrm{~kg}$} \\
\hline & Average & Std Dev & Average & Std Dev & Average & Std Dev & Average & Std Dev \\
\hline Adrenals & $1.75 E+01$ & $1.65 E+00$ & $1.52 E+01$ & $1.38 \mathrm{E}+00$ & $1.36 \mathrm{E}+01$ & $1.30 E+00$ & $1.23 E+01$ & $1.22 \mathrm{E}+00$ \\
\hline Brain & $1.33 E+01$ & $2.51 E+00$ & $1.14 \mathrm{E}+01$ & $2.13 E+00$ & $1.00 \mathrm{E}+01$ & $1.86 \mathrm{E}+00$ & $9.01 E+00$ & $1.67 E+00$ \\
\hline Breasts & $8.17 E+00$ & $1.22 \mathrm{E}+00$ & $7.05 E+00$ & $1.00 E+00$ & $6.23 E+00$ & $8.62 E-01$ & $5.63 E+00$ & $7.68 E-01$ \\
\hline Gallbladder wall & $1.65 E+02$ & $8.76 E+01$ & $1.48 \mathrm{E}+02$ & $8.19 \mathrm{E}+01$ & $1.43 \mathrm{E}+02$ & $8.02 E+01$ & $1.39 E+02$ & $7.86 \mathrm{E}+01$ \\
\hline LLI wall & $3.19 E+01$ & $1.09 \mathrm{E}+01$ & $2.96 \mathrm{E}+01$ & $1.05 E+01$ & $2.78 \mathrm{E}+01$ & $1.02 \mathrm{E}+01$ & $2.65 E+01$ & $9.91 E+00$ \\
\hline Small intestine & $7.10 E+01$ & $3.09 E+01$ & $6.81 E+01$ & $3.03 E+01$ & $6.55 E+01$ & $2.96 \mathrm{E}+01$ & $6.36 \mathrm{E}+01$ & $2.92 \mathrm{E}+01$ \\
\hline Stomach wall & $2.24 \mathrm{E}+01$ & $2.31 E+01$ & $1.31 E+01$ & $9.96 \mathrm{E}-01$ & $1.17 E+01$ & $8.99 E-01$ & $1.06 \mathrm{E}+01$ & $8.34 \mathrm{E}-01$ \\
\hline ULI wall & $7.52 E+01$ & $3.99 E+01$ & $7.80 E+01$ & $3.52 E+01$ & $7.44 \mathrm{E}+01$ & $3.42 E+01$ & $7.17 E+01$ & $3.33 E+01$ \\
\hline Heart wall & $1.72 E+01$ & $2.06 \mathrm{E}+00$ & $1.48 \mathrm{E}+01$ & $1.81 E+00$ & $1.27 E+01$ & $1.86 \mathrm{E}+00$ & $1.15 E+01$ & $1.67 \mathrm{E}+00$ \\
\hline Kidneys & $1.67 E+01$ & $1.45 E+00$ & $1.45 E+01$ & $1.17 E+00$ & $1.30 E+01$ & $1.11 E+00$ & $1.18 \mathrm{E}+01$ & $1.02 \mathrm{E}+00$ \\
\hline Liver & $8.59 E+01$ & $2.94 \mathrm{E}+01$ & $7.40 \mathrm{E}+01$ & $2.48 \mathrm{E}+01$ & $6.44 \mathrm{E}+01$ & $2.21 E+01$ & $5.77 E+01$ & $1.98 \mathrm{E}+01$ \\
\hline Lungs & $1.10 E+01$ & $9.93 E-01$ & $9.58 \mathrm{E}+00$ & $8.91 E-01$ & $8.52 E+00$ & $8.01 E-01$ & $7.73 E+00$ & $7.21 E-01$ \\
\hline Muscle & $1.11 \mathrm{E}+01$ & $1.06 \mathrm{E}+00$ & $9.70 \mathrm{E}+00$ & $8.76 \mathrm{E}-01$ & $8.62 \mathrm{E}+00$ & $7.59 \mathrm{E}-01$ & $7.82 \mathrm{E}+00$ & $6.79 E-01$ \\
\hline Ovaries & $2.20 E+01$ & $5.15 E+00$ & $1.97 E+01$ & $4.79 E+00$ & $1.76 \mathrm{E}+01$ & $4.32 E+00$ & $1.61 E+01$ & $3.96 \mathrm{E}+00$ \\
\hline Pancreas & $1.90 E+01$ & $2.91 E+00$ & $1.61 E+01$ & $1.42 \mathrm{E}+00$ & $1.44 \mathrm{E}+01$ & $1.35 E+00$ & $1.31 E+01$ & $1.24 \mathrm{E}+00$ \\
\hline Red marrow & $1.96 \mathrm{E}+01$ & $2.24 \mathrm{E}+00$ & $1.65 E+01$ & $1.96 \mathrm{E}+00$ & $1.43 \mathrm{E}+01$ & $1.76 \mathrm{E}+00$ & $1.27 E+01$ & $1.63 E+00$ \\
\hline Osteogenic cells & $4.01 E+01$ & $4.90 E+00$ & $3.27 E+01$ & $4.21 E+00$ & $2.76 E+01$ & $3.71 E+00$ & $2.40 E+01$ & $3.34 \mathrm{E}+00$ \\
\hline Skin & $7.75 E+00$ & $1.11 E+00$ & $6.70 E+00$ & $9.32 \mathrm{E}-01$ & $5.92 \mathrm{E}+00$ & $8.06 \mathrm{E}-01$ & $5.34 \mathrm{E}+00$ & $7.15 E-01$ \\
\hline Spleen & $1.19 E+01$ & $2.21 E+00$ & $1.00 E+01$ & $1.14 \mathrm{E}+00$ & $8.90 E+00$ & $9.80 E-01$ & $8.07 E+00$ & $8.85 E-01$ \\
\hline Testes & $8.84 \mathrm{E}+00$ & $1.70 E+00$ & $7.68 \mathrm{E}+00$ & $1.47 E+00$ & $6.81 E+00$ & $1.29 E+00$ & $6.16 \mathrm{E}+00$ & $1.16 \mathrm{E}+00$ \\
\hline Thymus & $9.44 \mathrm{E}+00$ & $1.64 \mathrm{E}+00$ & $8.19 E+00$ & $1.39 E+00$ & $7.26 \mathrm{E}+00$ & $1.21 E+00$ & $6.57 E+00$ & $1.09 E+00$ \\
\hline Thyroid & $8.79 E+00$ & $1.90 \mathrm{E}+00$ & $7.61 E+00$ & $1.62 E+00$ & $6.75 E+00$ & $1.43 \mathrm{E}+00$ & $6.10 E+00$ & $1.28 \mathrm{E}+00$ \\
\hline Urinary bladder wall & $3.10 E+01$ & $1.25 E+01$ & $2.88 \mathrm{E}+01$ & $1.21 E+01$ & $2.71 E+01$ & $1.17 E+01$ & $2.58 \mathrm{E}+01$ & $1.14 \mathrm{E}+01$ \\
\hline Uterus & $1.97 E+01$ & $3.84 \mathrm{E}+00$ & $1.74 \mathrm{E}+01$ & $3.55 E+00$ & $1.56 \mathrm{E}+01$ & $3.19 E+00$ & $1.42 \mathrm{E}+01$ & $2.96 \mathrm{E}+00$ \\
\hline Total body & $1.50 E+01$ & $1.14 \mathrm{E}+00$ & $1.30 E+01$ & $1.02 E+00$ & $1.16 E+01$ & $9.43 E-01$ & $1.05 E+01$ & $8.76 E-01$ \\
\hline Effective dose & $2.37 E+01$ & $5.50 \mathrm{E}+00$ & $2.06 \mathrm{E}+01$ & $4.55 E+00$ & $1.86 \mathrm{E}+01$ & $4.26 \mathrm{E}+00$ & $1.72 \mathrm{E}+01$ & $4.04 \mathrm{E}+00$ \\
\hline
\end{tabular}


Table 5 Comparison of ED (adult phantom model) estimates for florbetapir F 18 and other ${ }^{18} \mathrm{~F}$-labeled pharmaceuticals

\begin{tabular}{lc}
\hline Tracer & ED $(\mu \mathrm{Sv} / \mathrm{MBq})$ \\
\hline Florbetapir F 18 & 18.60 \\
Flutemetamol $\left[{ }^{18} \mathrm{~F}\right.$-GE067] [17] & 33.8 \\
Florbetaben $\left[{ }^{18} \mathrm{~F}-\mathrm{BAY} 94-9172\right][18]$ & 14.7 \\
${ }^{18} \mathrm{~F}-\mathrm{FDG}[15]$ & 19.0 \\
${ }^{11} \mathrm{C}-\mathrm{PIB}[18]$ & 5.29 \\
\hline
\end{tabular}

$18.5 \pm 4.23 \mu \mathrm{Sv} / \mathrm{MBq}$ or $6.84 \mathrm{mSv}$ for $370 \mathrm{MBq}$. Tables 2 and 3 summarizes average organ dosimetry for nine subjects with different urinary bladder voiding models. Data for both voiding models shows that the organs that received the highest radiation absorbed dose were the gallbladder, upper large intestine, small intestine, liver, and urinary bladder at $143.0 \pm 80.20,74.50 \pm 34.20$, $65.50 \pm 29.60,64.40 \pm 22.10$, and $27.10 \pm 11.70 \mu \mathrm{Sv} / \mathrm{MBq}$, respectively.

The organ volume, organ mass, and whole body mass were selected and scaled [15] by ratios to the 70-kg reference phantom model to obtain dose estimates for 50-, 60-, and 80-kg individuals. Average dose estimates for different body weights are summarized in Table 4.

\section{Discussion}

The present study was designed to provide the biodistribution and radiation dosimetry of florbetapir. Our study has resulted in an ED estimate of $18.6 \pm 4.26 \mu \mathrm{Sv} /$ $\mathrm{MBq}$ or $6.88 \mathrm{mSv}$ for $370 \mathrm{MBq}$ for PET. We found that florbetapir is rapidly distributed throughout the body shortly following I.V. administration. The gallbladder was the organ that received the highest absorbed dose, with an average value of $143.0 \pm 80.20 \mu \mathrm{Sv} / \mathrm{MBq}$ across nine healthy volunteers. Variability in gallbladder activity was observed, which is possibly related to differences in the individual kinetics of gallbladder emptying or differences across subjects in the timing of food consumption or diet. Images over time show that elimination occurs primarily by way of clearance from the liver and excretion through the gallbladder into the GI tract. Some accumulation is also observed in the urinary bladder. Modeling urinary bladder voiding at $90 \mathrm{~min}$ post-injection did not substantially change the radiation dosimetry results.

The present findings are very similar to the results of a preliminary study [16] that calculated an effective dose of $19.3 \pm 1.30 \mu \mathrm{Sv} / \mathrm{MBq}$ in three subjects from an Asian population and evaluated the organ kinetics over a shorter $(3 \mathrm{~h})$ time period. In the present study, the radiation dosimetry of florbetapir was studied in nine healthy volunteers from an American population and the organ kinetics were evaluated for approximately $6 \mathrm{~h}$. Radiation dosimetry of other ${ }^{18} \mathrm{~F}$-labeled PET amyloid tracers has been evaluated by a methodology similar to that of the present study. Koole et al. [17] and O'Keefe et al. [18] previously studied ${ }^{18} \mathrm{~F}$-GE067 (flutemetamol) and ${ }^{18}$ F-BAY94-9172 (florbetaben) radiation dosimetries, respectively. O'Keefe et al. [18] also reported the radiation dose estimate for the ${ }^{11} \mathrm{C}$-labeled PET amyloid tracer ${ }^{11} \mathrm{C}$-PIB. The International Commission on Radiological Protection (ICRP), in its Publication 106 [15], presents tables of ${ }^{18} \mathrm{~F}$-fluorodeoxyglucose $\left({ }^{18} \mathrm{~F}\right.$ FDG) and reports the effective dose. The ICRP authors derived a kinetic model based on the data published by Hays and Segall [19], Deloar et al. [20], and Meija et al. [21]. The retention in the specified source organs was considered to be infinite. The ED calculated for florbetapir, flutemetamol $\left[{ }^{18} \mathrm{~F}-\mathrm{GE} 067\right]$, florbetaben $\left[{ }^{18} \mathrm{~F}\right.$ BAY94-9172], ${ }^{18}$ F-FDG, and ${ }^{11} \mathrm{C}$-PIB are summarized in Table 5.

\section{Conclusions}

The radiation dosimetry for the ${ }^{18}$ F-labeled amyloid imaging agent florbetapir has been calculated in nine healthy volunteers and results in an effective radiation dose of $18.60 \pm 4.26 \mu \mathrm{Sv} / \mathrm{MBq}$ or $6.88 \mathrm{mSv}$ for $370 \mathrm{MBq}$.

\section{Competing interests}

Abhinay D. Joshi, Michael J. Pontecorvo, Alan P. Carpenter, Mark A. Mintun, and Daniel M. Skovronsky are employees of Avid Radiopharmaceuticals, Inc. Lee P. Adler has no relevant financial disclosures other than the clinical study contract with Avid for this work. Michael G. Stabin has no relevant financial disclosures other than a consultant with Avid for this work.

\section{Authors' contributions}

ADJ participated in the study design, image analysis, and data interpretation and drafted the manuscript. MJP was responsible for the study concept and design, data interpretation and review of the manuscript. LA carried out the data acquisition and participated in the scientific review. MGS performed the dosimetry analysis. APC was responsible for the administrative and material support, data interpretation and critical review of the manuscript. DMS supervised the study, provided administrative or material support and participated in the critical review. MAM participated in the data interpretation and critical review of the manuscript. All authors read and approved the final manuscript.

\section{Acknowledgements}

This study was funded by Avid Radiopharmaceuticals Inc., Philadelphia, PA, USA.

\section{Author details}

'Avid Radiopharmaceuticals, Inc., 3711 Market Street, 7th Floor, Philadelphia, PA 19104, USA. ${ }^{2}$ Adler Institute for Advanced Imaging, Jenkintown, PA 19046, USA. ${ }^{3}$ Department of Radiology and Radiological Sciences, Vanderbilt University, Nashville, TN 37235, USA. ${ }^{4}$ Department of Radiology, University of Pennsylvania, Philadelphia, PA 19104, USA.

Received: 21 August 2013 Accepted: 26 November 2013

Published: 8 January 2014 


\section{References}

1. The National Institute on Aging and Reagan Institute Working Group on Diagnostic Criteria for the Neuropathological Assessment Of Alzheimer's Disease: Consensus recommendations for the postmortem diagnosis of Alzheimer's disease. Neurobiol Aging 1997, 18:S1-S2.

2. Klunk WE, Engler H, Nordberg A, Wang Y, Blomqvist G, Holt DP, Bergström M, Savitcheva I, Huang GF, Estrada S, Ausén B, Debnath ML, Barletta J, Price JC, Sandell J, Lopresti BJ, Wall A, Koivisto P, Antoni G, Mathis CA, Långström $B J$ : Imaging brain amyloid in Alzheimer's disease with Pittsburg Compound-B. Ann Neurol 2004, 55:306-319.

3. Nordberg A, Rinne JO, Kadir A, Långström B: The use of PET in Alzheimer disease. Nat Rev Neurol 2010, 6(2):78-87.

4. Klunk WE, Wang Y, Huang GF, Debnath ML, Holt DP, Mathis CA: Uncharged thioflavin-T derivatives bind to amyloid-beta protein with high affinity and readily enter the brain. Life Sci 2001, 69(13):1471-1484.

5. Rowe CC, Villemagne VL: Brain amyloid imaging. J Nucl Med 2011, 52(11):1733. 1740

6. Choi SR, Golding G, Zhuang Z, Zhang W, Lim N, Hefti F, Benedum TE, Kilbourn MR, Skovronsky D, Kung HF: Preclinical properties of ${ }^{18} \mathrm{~F}-\mathrm{AV}-45$ : a PET agent for A $\beta$ plaques in the brain. J Nucl Med 2009, 50:1887-1894.

7. Wong DF, Rosenberg PB, Zhou Y, Kumar A, Raymont V, Ravert HT, Dannals RF, Nandi A, Brasić JR, Ye W, Hilton J, Lyketsos C, Kung HF, Joshi AD, Skovronsky DM, Pontecorvo MJ: In vivo imaging of amyloid deposition in Alzheimer's disease using the novel radioligand ${ }^{18} \mathrm{~F}-\mathrm{AV}$-45 (florbetapir F 18). J Nucl Med 2010, 51(6):913-920.

8. Clark CM, Schneider JA, Bedell BJ, Beach TG, Bilker WB, Mintun MA, Pontecorvo MJ, Hefti F, Carpenter AP, Flitter ML, Krautkramer MJ, Kung $H F$, Coleman RE, Doraiswamy PM, Fleisher AS, Sabbagh MN, Sadowsky $\mathrm{CH}$, Reiman EP, Zehntner SP, Skovronsky DM, AV45-A07 Study Group: Use of florbetapir-PET for imaging $\beta$-amyloid pathology. JAMA 2011, 305(3):275-283.

9. Clark CM, Pontecorvo MJ, Beach TG, Bedell BJ, Coleman RE, Doraiswamy PM, Fleisher AS, Reiman EM, Sabbagh MN, Sadowsky CH, Schneider JA, Arora A, Carpenter AP, Flitter ML, Joshi AD, Krautkramer MJ, Lu M, Mintun MA, Skovronsky DM, AV-45-A16 Study Group: Cerebral PET with florbetapir compared with neuropathology at autopsy for detection of neuritic amyloid- $\beta$ plaques: a prospective cohort study. Lancet Neurol 2012, 11(8):669-678.

10. Rohen JK, Yokochi C: Color Atlas of Anatomy: A Photographic Study of the Human Body. 3rd edition. New York: Igaku-Shoin; 1992.

11. Netter FH: Atlas of Human Anatomy. Dover: Ciba-Geigy Corporation; 1989.

12. Foster DM, Barrett PHR: Developing and testing integrated multicompartmental models to describe a single-input multiple-output study using the SAAM II software system. In Mathematical Modeling of Experimental Nutrition. Edited by Clifford AJ. New York: Plenum; 1998:59-78.

13. Stabin MG, Siegel JA: Physical models and dose factors for use in internal dose assessment. Health Phys 2003, 85:294-310.

14. Stabin MG, Sparks RB, Crowe E: OLINDA/EXM: the second-generation personal computer software for internal dose assessment in nuclear medicine. J Nucl Med 2005, 46:1023-1027.

15. International Commission on Radiological Protection: Radiation Dose to Patients from Radiopharmaceuticals. ICRP Publication 106: Addendum 3 to Publication 53. Oxford: Pergamon; 1998.

16. Lin KJ, Hsu WC, Hsiao IT, Wey SP, Jin LW, Skovronsky D, Wai YY, Chang HP, Lo CW, Yao CH, Yen TC, Kung MP: Whole-body distribution and brain PET imaging with $\left[{ }^{18} \mathrm{~F}\right] \mathrm{AV}-45$, a novel amyloid imaging agent-a pilot study. Nucl Med Biol 2010, 37:497-508.

17. Koole M, Lewis D, Buckley C, Nelissen N, Vandenbulcke M, Brooks D, Vandenberghe $\mathrm{R}$, Van Laere K: Whole-body biodistribution and radiation dosimetry of ${ }^{18} \mathrm{~F}$-GE067: a radioligand for in vivo brain amyloid imaging. J Nucl Med 2009, 50:818-822.

18. O'Keefe G, Saunder TH, Steven Ng, Ackerman UWE, Tochon-Danguy HJ, Chan GJ, Gong S, Dyrk T, Lindemann S, Holl G, Dinkelborg L, Villemagne V, Rowe CC: Radiation dosimetry of $\beta$-amyloid tracers ${ }^{11} \mathrm{C}$-PIB and ${ }^{18} \mathrm{~F}-\mathrm{BAY} 94-9172$. J Nucl Med 2009, 50:309-315.

19. Hays MT, Segall GM: A mathematical model for the distribution of fluorodeoxyglucose in humans. J Nucl Med 1999, 40:1358-1366.

20. Deloar HM, Fujiwara T, Shidahara M, Nakamura T, Watabe H, Narita Y, Itoh M, Miyake M, Watanuki S: Estimation of absorbed dose for 2-[F-18] fluoro-2-deoxy-D-glucose using whole-body positron emission tomography and magnetic resonance imaging. Eur J Nucl Med 1998, 25:565-574.

21. Mejia AA, Nakamura T, Masatoshi I, Hatazawa J, Masaki M, Watanuki S: Estimation of absorbed doses in humans due to intra-venous administration of fluorine-18-fluorodeoxyglucose in PET studies. J Nucl Med 1991, 32:699-706.

doi:10.1186/2191-219X-4-4

Cite this article as: Joshi et al.: Radiation dosimetry of florbetapir F 18. EJNMMI Research 2014 4:4.

\section{Submit your manuscript to a SpringerOpen ${ }^{\odot}$ journal and benefit from:}

- Convenient online submission

- Rigorous peer review

- Immediate publication on acceptance

- Open access: articles freely available online

- High visibility within the field

- Retaining the copyright to your article

Submit your next manuscript at $>$ springeropen.com 